\section{Prevalence and associations of epiretinal membranes in an adult Japanese population: the Funagata study}

R Kawasaki 1,2,3, JJ Wang ${ }^{2,4}$, H Sato ${ }^{1}$, P Mitchell ${ }^{4}$, T Kato ${ }^{5,6}$, S Kawata ${ }^{5,7}$, T Kayama ${ }^{5,8}$, H Yamashita ${ }^{1,5}$ and TY Wong ${ }^{2,9}$

Keywords: epiretinal membrane; diabetes; population-based study; Funagata study; Japanese; prevalence

risk factors of epiretinal membranes (ERM) in a Japanese population.

Patients and Methods The Funagata Study examined 1758 Japanese aged 35 years or older (42\% of eligible) from June 2000 to June 2002. A total of $1723(98.0 \%)$ participants had nonmydriatic fundus photographs of the right eye to grade the presence of ERM, using the Blue Mountains Eye Study (BMES) protocol. After age standardization, the prevalence of ERM in the right eyes of the participants included in this study was compared with that reported from right eyes of participants in the BMES and the Beaver Dam Eye Study (BDES). ERM detected in eyes with diabetic retinopathy and other retinal lesions were excluded from the analysis of associations. Multiple logistic regression models were used to determine risk factors for the presence of ERM, adjusting for age and gender.

Results There were 84 right eyes with ERM, representing $5.44 \%$ of this sample. After age standardization, the prevalence rate $(5.7 \%)$ was within the range reported in the BMES (3.5\%) and the BDES $(6.9 \%)$. Older age (genderadjusted odds ratio (OR) per 10 years: $1.72,95 \%$ confidence interval (CI): 1.40-2.11) and diabetes (age-gender-adjusted OR: 1.84, 95\% CI: 1.01-3.37) were the only two factors associated with ERM. Conclusions We found a similar prevalence of ERM in the Japanese as in mainly white populations. Increasing age and diabetes were risk factors for ERM in this adult Japanese population.

Eye (2009) 23, 1045-1051; doi:10.1038/eye.2008.238; published online 8 August 2008
Department of Ophthalmology an Visual Science, Yamagata University Faculty of Medicine, Yamagata, Japan

${ }^{2}$ Centre for Eye Research Australia, University of Melbourne,

Victoria, Australia

${ }^{3}$ Department of Medicine, Centre for Clinical Research Excellence in Clinical Science in Diabetes, St Vincent's Hospital Melbourne, Victoria, Australia

${ }^{4}$ Centre for Vision Research, Westmead Millennium Institute, University of Sydney, NSW, Australia

${ }^{5}$ Yamagata University 21st Century COE program study group, Yamagata University Faculty of Medicine,

Yamagata, Japan

${ }^{6}$ Departments of Neurology، Hematology, Metabolism, Endocrinology and Diabetology, Yamagata University Faculty of Medicine, Yamagata, Japan

'Department of Gastroenterology, Yamagata University Faculty of Medicine, Yamagata, Japan

${ }^{8}$ Department of Neurosurgery, Yamagata University Faculty of Medicine, Yamagata, Japan

${ }^{9}$ Singapore Eye Research Institute, National University of Singapore, Singapore, Singapore

Correspondence:

R Kawasaki,

Department of Ophthalmology and Visual Science,

Yamagata University,

2-2-2 lida-Nishi,

Yamagata, Japan, 990-9585

Tel: + 8123628 5374;

Fax: + 81236285376

E-mail: ryok@

med.id.yamagata-u.ac.jp

Received: 7 March 2008 Accepted in revised form: 1 July 2008

Published online: 8 August 2008 
Table 1 Summary of previously reported characteristics (ORs and 95\% CIs) associated with ERM lesions in population-based epidemiologic studies

\begin{tabular}{|c|c|c|c|c|c|}
\hline \multicolumn{2}{|l|}{$C M R$} & \multicolumn{2}{|l|}{$P M F$} & \multicolumn{2}{|l|}{$E R M$} \\
\hline \multicolumn{2}{|c|}{ BMES $^{10}(\mathrm{n}=3654$, Australia, $1992-94)$} & \multicolumn{2}{|c|}{$B_{M M S^{10}}(\mathrm{n}=3654$, Australia, 1992-94) } & \multicolumn{2}{|c|}{$B D E S^{9}(\mathrm{n}=4926, U S A, 1988-90)$} \\
\hline Early ARM & $0.30(0.09,0.94)$ & LogMar visual acuity & $0.95(0.93,0.98)$ & Nuclear sclerotic cataract & $0.58(0.43,0.77)$ \\
\hline \multirow{4}{*}{$\begin{array}{l}\text { Refractive error } \\
\text { (dioptres) }\end{array}$} & $1.13(1.02,1.24)$ & Plasma glucose $(\mathrm{mmol} / \mathrm{l})$ & $1.20(1.07,1.34)$ & Cortical cataract & $0.65(0.49,0.86)$ \\
\hline & & Diabetes (history) & $2.68(1.05,6.86)$ & Any ARM & $0.69(0.53,0.90)$ \\
\hline & & $\begin{array}{l}\text { Diabetes (history or } \\
\text { blood test) }\end{array}$ & $3.21(1.43,7.23)$ & Early ARM & $0.76(0.58,1.00)$ \\
\hline & & & & Arteriovenous nicking & $1.80(1.00,3.25)$ \\
\hline \multicolumn{2}{|c|}{$\begin{array}{l}\text { Visual Impairment Project Study }{ }^{7}(\mathrm{n}=3271 \text {, } \\
\text { Australia, 1992-97) }\end{array}$} & \multicolumn{2}{|c|}{$\begin{array}{l}\text { Visual Impairment Project Study }(\mathrm{n}=3271 \text {, } \\
\text { Australia, 1992-97) }\end{array}$} & \multicolumn{2}{|c|}{$\begin{array}{l}\text { Visual Impairment Project Study }(\mathrm{n}=3271 \\
\text { Australia, 1992-97) }\end{array}$} \\
\hline \multirow[t]{3}{*}{ Visual acuity $(<6 / 6)$} & $1.5(1.1,2.0)$ & Visual acuity $(<6 / 6)$ & $1.9(1.0,3.6)$ & Smoking & $0.6(0.5,0.9)$ \\
\hline & & Myopia & $2.2(1.0,4.6)$ & Visual acuity $(6 / 6)$ & $1.6(1.2,2.2)$ \\
\hline & & Urban & $3.1(1.3,7.0)$ & & \\
\hline \multicolumn{2}{|c|}{ Beijing Eye Study $(\mathrm{n}=4439$, China, 2001) } & \multicolumn{2}{|c|}{ Beijing Eye Study $(\mathrm{n}=4439$, China, 2001) } & \multicolumn{2}{|c|}{ Hisayama Study $(\mathrm{n}=1775, \text { Japan, 1998 })^{6}$} \\
\hline Visual field loss & $(1.01,1.07)^{\mathrm{a}}$ & Visual field loss & $(1.02,1.08)^{\mathrm{a}}$ & $\begin{array}{l}\text { Serum cholesterol } \\
(\mathrm{mmol} / \mathrm{l})\end{array}$ & $1.29(1.00,1.68)$ \\
\hline \multirow{7}{*}{$\begin{array}{l}\text { Higher level of } \\
\text { education }\end{array}$} & $(1.26,1.92)^{\mathrm{a}}$ & Higher level of education & $(1.15,1.74)^{\mathrm{a}}$ & & \\
\hline & & & & \multicolumn{2}{|c|}{ Beijing Eye Study $(\mathrm{n}=4439, \text { China, 2001 })^{8}$} \\
\hline & & & & Hyperopic refractive error & $(1.01,1.18)^{\mathrm{a}}$ \\
\hline & & & & Visual field loss & $(1.03,1.07)^{\mathrm{a}}$ \\
\hline & & & & Higher level of education & $(1.29,1.74)^{\mathrm{a}}$ \\
\hline & & & & \multirow{2}{*}{\multicolumn{2}{|c|}{$\begin{array}{l}\text { Los Angeles Latino Eye Study }{ }^{11} \text { ( } \mathrm{n}=5982, \text { USA, } \\
\text { 2000-03) } \\
\text { Early ARM, high intraocular pressure, } \\
\text { diabetes, hypertension, smoking, and } \\
\text { refractive error were not significantly } \\
\text { associated with ERM }\end{array}$}} \\
\hline & & & & & \\
\hline
\end{tabular}

Abbreviations: $\mathrm{BDES}=$ Beaver Dam Eye Study; BMES=Blue Mountains Eye Study; $\mathrm{CI}=$ confidence interval; CMR=cellophane macular reflex; $\mathrm{ERM}=$ epiretinal membrane; $\mathrm{OR}=$ odds ratio; $\mathrm{PMF}=$ preretinal macular fibrosis.

All ORs are adjusted for age and/or gender except for those specified.

${ }^{\text {anN }}$ adjusted for age or gender.

suggested that the higher prevalence in Hispanics as compared with other racial groups may be related to the higher frequency of diabetes in this racial/ethnic group. ${ }^{9}$

In this report, we aim to describe the age- and genderspecific prevalence of ERM and associated risk factors in an adult Japanese population and to compare the prevalence rates with two other mainly white populations (the Blue Mountains Eye Study (BMES) in Australia and the Beaver Dam Eye study (BDES) in Wisconsin, USA) after age standardization to the world standard population.

\section{Methods}

\section{Study population}

This study was conducted as part of the Funagata Study, a population-based cohort study of adult Japanese between June 2000 and June 2002. The study population and research methodology have been described in detail earlier. ${ }^{12}$ In brief, 4160 residents aged 35 years or older in Funagata town, Yamagata, Japan, were identified. After excluding 484 with severe disabilities or with diabetes, and under treatment, 3676 residents were considered eligible. Of those, 1961 were examined in the study, and 1758 had a detailed ophthalmic examination $(42.3 \%$ of eligible participants). Of these, 1723 (98.0\% of 1758) had retinal photographs of satisfactory quality to assess ERM lesions, and were included in our analyses. This study was conducted according to the recommendations of the Declaration of Helsinki and was approved by the Institutional Review Board at the Yamagata University School of Medicine, Yamagata, Japan. Written consent was obtained from the study participants.

\section{Fundus photography}

A fundus photograph of the right eye was obtained using a $45^{\circ}$ non-mydriatic fundus camera (CR5-NM45, Canon Inc. Tokyo, Japan and TRC-NW, Topcon Inc. Tokyo, 
Japan) on a 35-mm slide film. The image was centred on the region between the optic disc and macula. Pupil dilation was achieved after $5 \mathrm{~min}$ in a dark room without using pharmacologic dilating agents. If the fundus photograph of the right eye could not be performed with adequate quality, fundus photography of the left eye was performed.

\section{Grading of retinal photographs and definitions of ERM}

All fundus photographs were evaluated at the Image Reading Centre, Centre for Vision Research (Sydney, Australia) by trained graders, using a standardized grading approach for retinal lesions, masked to participants' information. The definition of ERM lesions used in BMES ${ }^{10}$ was originally adopted from BDES. ${ }^{11}$ Two stages of ERM lesions were identified: ${ }^{11}$ a less severe form termed 'cellophane macular reflex' (CMR) was defined if 'glinting, water silk, and shifting light reflex' was present without visible retinal folds; ${ }^{1,10}$ and a more severe form termed 'preretinal macular fibrosis' (PMF) was defined if retinal folds were present, with a 'more opaque, greyish appearance on the inner retinal surface ${ }^{11}$ The site of the epiretinal membrane was classified using a grid with a radius of $3000 \mu \mathrm{m}$, corresponding to the anatomic macula. Epiretinal membranes outside the grid were graded as not present. ${ }^{10}$ The term ERM was defined to include participants with either CMR or PMF. All ERM signs were adjudicated by a senior researcher (JJW) or retinal specialist (PM).

If ERM and other retinal diseases coexisted within the same eye, they were classified as 'secondary' ERM, and are excluded from the analysis of associations in this report. Retinal diseases other than ERM were also assessed by the grader using similar standardized approaches. Signs of diabetic retinopathy (microaneurysms, retinal haemorrhages, exudates, or proliferative retinopathy) were graded with the modified Airlie House Classification, as used in the Early Treatment Diabetic Retinopathy study; ${ }^{13}$ early agerelated maculopathy and age-related macular degeneration were defined following the modification of the Wisconsin Age-Related Maculopathy Grading System $^{14}$ as used in the BMES; branch retinal vein occlusion and central retinal vein occlusion were assessed as present or absent. All retinal pathology was adjudicated by a retinal specialist (PM). ERM cases considered secondary to these retinal conditions were excluded from the analysis, but coexisting early agerelated maculopathy lesions were included in the primary analysis, as early age-related maculopathy signs do not lead to secondary ERM.

\section{Systemic assessment}

Details of the systemic assessment are provided elsewhere. ${ }^{12}$ In summary, blood pressure was measured after participants were seated comfortably for at least $5 \mathrm{~min}$. A single measure of systolic blood pressure and diastolic blood pressure was used. Hypertension status was defined using the 2003 World Health Organization (WHO) guidelines; ${ }^{15}$ people with hypertension stage I to stage III (systolic blood pressure $\geqslant 140 \mathrm{~mm} \mathrm{Hg}$ or diastolic blood pressure $\geqslant 90 \mathrm{~mm} \mathrm{Hg}$ ) or previous diagnosis of hypertension were defined as having 'hypertension'. Diabetes and glucose tolerance status were defined on the basis of the results of a $75 \mathrm{~g}$ oral glucose tolerance test following WHO guidelines. ${ }^{16}$ 'Pre-diabetes' was defined as either impaired glucose tolerance (fasting plasma glucose $<126 \mathrm{mg} / 100 \mathrm{ml}$ $(7.0 \mathrm{mmol} / \mathrm{l})$, and $2 \mathrm{~h}$ post-load glucose $\geqslant 140 \mathrm{mg} / 100 \mathrm{ml}$ (7.8 mmol/1) but $<200 \mathrm{mg} / 100 \mathrm{ml}(11.1 \mathrm{mmol} / \mathrm{l})$ ), or impaired fasting glucose (fasting plasma glucose $\geqslant 110 \mathrm{mg} / 100 \mathrm{ml}(6.1 \mathrm{mmol} / 100 \mathrm{ml})$ but $<126 \mathrm{mg} / 100 \mathrm{ml}$ $(7.0 \mathrm{mmol} / \mathrm{l})$ and $2 \mathrm{~h}$ post-load glucose $<140 \mathrm{mg} / 100 \mathrm{ml}$ $(7.8 \mathrm{mmol} / \mathrm{l}))$. Total cholesterol, triglyceride, and highdensity lipoprotein cholesterol were also measured. Selfreported smoking status was assessed. Body mass index was calculated as weight $(\mathrm{kg})$ divided by the square of height $(\mathrm{m})$.

\section{Data analysis}

The age- and gender-specific prevalence of CMR, PMF, and any ERM (CMR or PMF) were assessed. The MannWhitney $U$-test or $\chi^{2}$-test was used to compare demographic characteristics. Direct age standardization of our sample, the BMES and BDES populations to the WHO world standard population, ${ }^{17}$ was performed to enable comparison of ERM prevalence across the three study samples. Logistic regression models were used to assess associations with idiopathic ERM. Potential associations included age (per 10 years), gender (female vs male), systolic and diastolic blood pressure (per $10 \mathrm{~mm} \mathrm{Hg}$ ), hypertension (present $v$ s absent), body mass index (per $1 \mathrm{~kg} / \mathrm{m}^{2}$ ), total cholesterol (per $10 \mathrm{mg} / 100 \mathrm{ml}$ ), triglyceride (per $10 \mathrm{mg} / 100 \mathrm{ml}$ ), HDL cholesterol (per $10 \mathrm{mg} / 100 \mathrm{ml}$ ), Smoking status (current smoker vs past or never smoker), fasting plasma glucose (per $10 \mathrm{mg} /$ $100 \mathrm{ml}$ ), diabetes status (pre-diabetes or diabetes vs nondiabetes), and early age-related maculopathy (present $v$ s absent). Participants $(n=180)$ with the following retinal diseases other than ERM were excluded from the logistic regression analyses for idiopathic ERM: diabetic retinopathy $(n=171)$, late age-related macular degeneration $(n=8)$, branch or central retinal vein occlusion $(n=10)$, and 9 of those overlapping owing to 
the coexistence of two conditions. Stata software (Stata 9.2 for Windows, StataCorp Inc. College Station, Texas, USA) was used for statistical analysis. Odds ratios (OR) and $95 \%$ confidence intervals (CI) are presented.

\section{Results}

\section{Demographic characteristics of the population}

Participants included in the analyses were significantly younger than those excluded because of poor photograph quality (mean age 60.6 years vs 69.4 years, $P<0.001$ ). There were no differences in systolic or diastolic blood pressure, body mass index, serum lipids, smoking status, hypertension, and diabetes status between the two groups (data not shown).

\section{Prevalence of ERM}

The overall prevalence of idiopathic CMR, PMF, and any ERM in the right eye was $3.95,1.49$, and $5.44 \%$, respectively. All of these rates increased with age (all $P$ for trend $P<0.001)$ and there was a similar prevalence on any idiopathic ERM in men $(n=35,5.2 \%)$, and women $(n=49,5.6 \%)$. There were also no significant gender differences in the prevalence of CMR or PMF separately (all $P>0.1$ ). Table 2 presents the age- and gender-specific prevalence of idiopathic ERM lesions in right eyes.

Table 2 Prevalence of CMR, PMF, and any ERM (any ERM) in the right eyes: the Funagata Study, Japan 2000-02

\begin{tabular}{|c|c|c|c|c|c|c|c|}
\hline \multirow[t]{2}{*}{ Age groups (yrs) } & \multirow[t]{2}{*}{ No. at risk } & \multicolumn{2}{|c|}{$C M R$} & \multicolumn{2}{|c|}{$P M F$} & \multicolumn{2}{|c|}{$E R M$} \\
\hline & & Eye & $(\%)$ & Eye & $(\%)$ & Eye & $(\%)$ \\
\hline \multicolumn{8}{|l|}{ Men } \\
\hline$<54$ & 252 & 3 & 1.19 & 2 & 0.79 & 5 & 1.98 \\
\hline $55-64$ & 133 & 4 & 3.01 & 1 & 0.75 & 5 & 3.76 \\
\hline $65-74$ & 184 & 16 & 8.70 & 3 & 1.63 & 19 & 10.33 \\
\hline$>75$ & 101 & 4 & 3.96 & 2 & 1.98 & 6 & 5.94 \\
\hline Total & 670 & 27 & 4.03 & 8 & 1.19 & 35 & 5.22 \\
\hline \multicolumn{8}{|l|}{ Women } \\
\hline$<54$ & 320 & 3 & 0.94 & 0 & 0.00 & 3 & 0.94 \\
\hline $55-64$ & 182 & 7 & 3.85 & 4 & 2.20 & 11 & 6.05 \\
\hline $65-74$ & 267 & 15 & 5.62 & 6 & 2.25 & 21 & 7.87 \\
\hline$>75$ & 104 & 9 & 8.65 & 5 & 4.81 & 14 & 13.46 \\
\hline Total & 873 & 34 & 3.89 & 15 & 1.72 & 49 & 5.61 \\
\hline \multicolumn{8}{|l|}{ Total } \\
\hline$<54$ & 572 & 6 & 1.05 & 2 & 0.35 & 8 & 1.40 \\
\hline $55-64$ & 315 & 11 & 3.49 & 5 & 1.59 & 16 & 5.08 \\
\hline $65-74$ & 451 & 31 & 6.87 & 9 & 2.00 & 40 & 8.87 \\
\hline$>75$ & 205 & 13 & 6.34 & 7 & 3.41 & 20 & 9.75 \\
\hline Total & 1543 & 61 & 3.95 & 23 & 1.49 & 84 & 5.44 \\
\hline
\end{tabular}

Abbreviations: $\mathrm{CMR}=$ cellophane macular reflex; $\mathrm{ERM}=$ epiretinal membrane; $\mathrm{PMF}=$ preretinal macular fibrosis.
Secondary ERM was found in 10 (5.6\%) of 180 participants; all of those were with coexisting diabetic retinopathy.

Table 3 presents the age-standardized prevalence of idiopathic CMR, PMF, and any ERM in the right eyes of the Funagata, BMES, and BDES study populations. After age standardization to the world standard population, ERM prevalence in our study $(5.7 \%$, CI $4.5-7.0 \%)$ was between the corresponding prevalence from the BMES (3.5\%, CI 2.9-4.1\%) and BDES (6.9\%, CI 6.1-7.7\%).

\section{Risk factor associations with ERM}

Increasing age was associated with an increased likelihood of idiopathic ERM (OR 1.72, CI 1.40-2.11, per 10 -year increase) after adjusting for gender. In crude analyses, higher levels of HDL cholesterol, fasting plasma glucose, and the presence of diabetes (without diabetic retinopathy) or early ARM were also associated with an increased ERM prevalence. After adjusting for age and gender, only the presence of diabetes was significantly associated with ERM (OR 1.84, CI 1.01-3.37) (Table 4). We repeated logistic regression analysis separately for persons with or without diabetes, with similar findings. Increasing age was consistently associated with ERM in both groups, but other factors were not associated with ERM after adjusting for age and gender.

\section{Discussion}

In this Japanese population-based study, we documented an overall ERM prevalence (based on data from right eyes only) of $5.44 \%$, including $3.95 \%$ with CMR and $1.49 \%$ with PMF. In contrast to the previous report from another Japanese population, ${ }^{6}$ we found a relatively similar age-standardized prevalence of ERM to that reported mainly from Caucasian white populations.

The Hisayama Study report previously suggested that ERM was less frequent among the Japanese than among the Caucasian population samples, compared with that in the BMES and BDES. ${ }^{6}$ The proposed reasons for the lower Hisayama Study Japanese prevalence included differences in methodology, such as the use of different fundus cameras and protocols (a $45^{\circ}$ non-mydriatic fundus camera was used in the Hisayama Study, compared with $30^{\circ}$ stereo fundus photography used in the BMES and BDES). Our study, with age standardization and improved comparability because of the assessment in a grading center using the same protocols, graders, and facilities as the BMES, however, does not support this previous observation. We therefore feel that there are likely to be other factors explaining the difference in the prevalence of ERM between our study 
Table 3 Age-specific prevalence and age-standardized prevalence of ERM lesions in the Funagata Study (Japan, 2000-02) compared with data from the BMES (Australia, 1992-93) and the BDES (USA, 1988-90)

\begin{tabular}{llcc}
\hline Study & Country, year & Fundus photography & Age-standardized prevalence $(\%)(95 \%$ CI) \\
\hline CMR & & & $4.1(3.0-5.2 \%)$ \\
Funagata study & Japan, 2000-02 & $45^{\circ} \mathrm{NM}$ & $2.8(2.3-3.3 \%)$ \\
BMES & Australia, 1992-93 & $30^{\circ} \mathrm{ST}$ & $5.3(4.6-6.1 \%)$ \\
BDES & USA, 1988-90 & $30^{\circ} \mathrm{ST}$ & $1.6(0.9-2.3 \%)$ \\
PMF & & $0.7(0.5-1.0 \%)$ \\
Funagata study & Japan, 2000-02 & $45^{\circ} \mathrm{NM}$ & $1.6(1.2-2.0 \%)$ \\
BMES & Australia, 1992-93 & $30^{\circ} \mathrm{ST}$ & \\
BDES & USA, 1988-90 & $30^{\circ} \mathrm{ST}$ & $5.7(4.5-7.0 \%)$ \\
ERM & & & $3.5(2.9-4.1 \%)$ \\
Funagata study & Japan, 2000-02 & $45^{\circ} \mathrm{NM}$ & $6.9(6.1-7.7 \%)$ \\
BMES & Australia, 1992-93 & $30^{\circ} \mathrm{ST}$ & $30^{\circ} \mathrm{ST}$ \\
BDES & USA, 1988-90 & & \\
\hline
\end{tabular}

Abbreviations: BDES = Beaver Dam Eye Study; BMES = Blue Mountains Eye Study; CMR = cellophane macular reflex; ERM=epiretinal membrane; $\mathrm{NM}=$ non-mydriatic fundus photography; $\mathrm{PMF}=$ preretinal macular fibrosis; $\mathrm{ST}$ = stereo fundus photography.

${ }^{\mathrm{a}} \mathrm{Age}$ standardized to $\mathrm{WHO}$ world standard population. ${ }^{18}$

Table 4 Associations of idiopathic ERM to selected risk factors adjusted for age and gender: the Funagata study (Japan, 2000-02)

\begin{tabular}{lcc}
\hline Characteristics & $\begin{array}{c}\text { Crude } \\
\text { OR }(95 \% \text { CI })\end{array}$ & $\begin{array}{c}\text { Age- and gender-adjusted } \\
\text { OR (95\% CI) }\end{array}$ \\
\hline Age (per 10 years) & $1.68(1.37,2.05)^{* *}$ & $1.72(1.40,2.11)^{* *, a}$ \\
Gender (female vs male) & $1.08(0.69,1.69)$ & $1.14(0.72,1.79)^{\mathrm{b}}$ \\
Systolic blood pressure (per 10 mm Hg) & $1.11(0.98,1.25)$ & $1.02(0.89,1.17)$ \\
Diastolic blood pressure (per 10 mm Hg) & $1.06(0.85,1.32)$ & $1.01(0.80,1.28)$ \\
Hypertension (present vs absent) & $1.69(1.09,2.61)^{*}$ & $1.15(0.73,1.81)$ \\
Body mass index & $0.99(0.93,1.05)$ & $1.00(0.94,1.07)$ \\
Total cholesterol (per 10 mg/100 ml) & $1.04(0.98,1.11)$ & $1.02(0.96,1.09)$ \\
Triglyceride (per 10 mg/100 ml) & $0.98(0.95,1.02)$ & $0.98(0.95,1.02)$ \\
HDL cholesterol (per 10 mm Hg) & $1.15(1.00,1.33)^{*}$ & $1.14(0.99,1.32)$ \\
Fasting plasma glucose (per 10 mm Hg) & $1.07(1.01,1.13)^{*}$ & $1.06(1.00,1.13)$ \\
Pre-diabetes (vs non-diabetes) & $1.61(0.98,2.68)$ & $1.34(0.80,2.23)$ \\
Diabetes excluding persons with retinopathy (vs non-diabetes) & $2.34(1.30,4.21)^{* *}$ & $1.84(1.01,3.37)^{*}$ \\
Smoking (current smoker $v s$ past or never smoker) & $0.58(0.30,1.13)$ & $0.78(0.37,1.63)$ \\
Early age-related maculopathy (present $v s$ absent) & $2.88(1.25,6.60)^{*}$ & $2.10(0.90,4.90)$ \\
\hline
\end{tabular}

Abbreviations: ERM, epiretinal membrane; $\mathrm{CI}$, confidence interval; OR, odds ratio.

${ }^{*} P<0.05 ;{ }^{*} P<0.001$.

${ }^{\mathrm{a} G e n d e r-a d j u s t e d ~ O R}$ is shown.

${ }^{\mathrm{b}}$ Age-adjusted OR is shown.

and the Hisayama Study, such as differences in grading definition and grading procedures between the two studies, for which we cannot assess from our study alone.

Our data indicate that diabetes (in the absence of clinical retinopathy) is an important systemic risk factor associated with ERM, independent of age (OR 1.84). Assuming that $10 \%$ of the population are affected with diabetes in Japan, ${ }^{18} 7.8 \%$ of ERM may be attributable to diabetes in the Japanese population. Our data thus suggest that diabetes is an important risk factor for ERM, even without diabetic retinopathy. Although increasing age was consistently found to be associated with ERM in previous population-based studies, ${ }^{6,7,9-11}$ the association between diabetes (after excluding persons without retinopathy) and ERM in population-based studies has been inconsistent. This association was mainly reported from a white population such as the BMES, where a history of diabetes was associated with PMF, but not with CMR or any ERM (Table 1). The Los Angeles Latino Eye Study also documented a significant association between diabetes and ERM. These authors speculated that the high prevalence of ERM in their Latino population was because of its high prevalence of diabetes. ${ }^{9}$ In a large 
ERM case series, ${ }^{19}$ persons with diabetes were documented to be more likely to have bilateral involvement with idiopathic ERM than those without diabetes

The association between diabetes and ERM might be explained by vitreous changes among persons with diabetes, as this is associated with a greater frequency of vitreous synchysis, even in the absence of retinopathy. ${ }^{20}$ Alteration of vitreous collagen and subsequent vitreous liquefaction have been suggested to induce $P V D,{ }^{21}$ and a very high proportion of ERM cases (78-100\%) was found to have either complete or partial PVD. ${ }^{2,3,22,23}$ PVD has thus been considered a factor involved in the genesis of ERM. $^{22,23}$ Alternatively, vitreous liquefaction might be directly involved in the development of ERM, as a remnant of the posterior wall of a premacular liquefied vitreous pocket was found to be associated with ERM. ${ }^{4}$ Various cytokines have also reportedly been involved in ERM. ${ }^{24}$ In most previous studies, the involvement of cytokines was mainly described in secondary ERM cases with proliferative diabetic retinopathy or other retinal diseases, so that the findings from such studies may not apply to idiopathic ERM, even though some of the same cytokines are suggested to be involved in idiopathic ERM formation. ${ }^{24}$

Although hypercholesterolaemia was related to ERM in the Japanese Hisayama Study, ${ }^{6}$ we could not find an association between total cholesterol level and ERM. Although early age-related maculopathy was reported to be negatively associated with $\mathrm{CMR}^{10}$ or ERM, ${ }^{11}$ this was not confirmed in our study, which showed a positive crude association between early age-related maculopathy and ERM, but was nonsignificant after adjusting for age and gender.

Limitations and potential biases should be noted. First, our study response rate was only moderate. This could have caused some selection bias resulting in either under- or overestimation of ERM prevalence and its associations. The participants included in our analysis were younger than those excluded. Second, only one fundus photograph of each participant was taken in our study. As only $19-31 \%$ of ERM cases were reported to have bilateral lesions, ${ }^{7,9-11}$ we could have overlooked a substantial proportion of ERM cases by using the information from only one eye. These factors may have led us to underestimate the overall ERM prevalence, though our prevalence is relatively similar to that from other population-based studies, after age standardization. Third, comparison between different populations could also be limited owing to differences in resolution of the various fundus cameras used. Although ERM lesions were assessed using the same grading protocols and facilities as used in BMES, fundus photography was performed using a non-mydriatic non-stereo camera. If stereo fundus photography improved the detection of ERM lesions when compared with non-mydriatic fundus photography, our study again could have underestimated the prevalence of ERM compared with other studies using stereo fundus photography. Finally, we could not assess all potential ERM risk factors, including eye conditions related to ERM, such as past history of cataract surgery. ${ }^{25}$ We could also have overlooked other possible conditions in the peripheral retina leading to secondary ERM, because of our photography in only one field at the posterior pole. Therefore, we could have underestimated the prevalence of secondary ERM or overestimated the prevalence of primary ERM. The strength of our study is that we have improved the comparability of our study findings to the BMES and BDES by using right eye data only from all three studies and age standardizing to a reference population.

In conclusion, we describe the age- and gender-specific prevalence of ERM lesions in an adult Japanese population. Our study finds a relatively similar ERM prevalence to that reported from two mainly white populations (BMES and the BDES). In addition to age, diabetes was associated with idiopathic ERM in this older Japanese population.

\section{Acknowledgements}

Source of funding: This study was supported by the 21st Century Center of Excellence (COE) project no.F03 ('Molecular Epidemiological Study utilizing the Regional Characteristics', Yamagata University) funded by Japanese Society for the Promotion of Science.

Presentation: Part of this paper was presented at the annual meeting of the Association for Research in Vision and Ophthalmology in 2006 (Fort Lauderdale, FL, USA).

\section{Conflict of interest/proprietary interest}

None.

\section{References}

1 Wise GN. Clinical features of idiopathic preretinal macular fibrosis. Schoenberg Lecture. Am J Ophthalmol 1975; 79: 349-347.

2 Hirokawa H, Jalkh AE, Takahashi M, Trempe CL, Schepens CL. Role of the vitreous in idiopathic preretinal macular fibrosis. Am J Ophthalmol 1986; 101: 166-169.

3 Appiah AP, Hirose T, Kado M. A review of 324 cases of idiopathic premacular gliosis. Am J Ophthalmol 1988; 106: 533-535.

4 Kishi S, Shimizu K. Oval defect in detached posterior hyaloid membrane in idiopathic preretinal macular fibrosis. Am J Ophthalmol 1994; 118: 451-456.

5 Sidd RJ, Fine SL, Owens SL, Patz A. Idiopathic preretinal gliosis. Am J Ophthalmol 1982; 94: 44-48. 
6 Miyazaki M, Nakamura H, Kubo M, Kiyohara Y, Iida M, Ishibashi $\mathrm{T}$ et al. Prevalence and risk factors for epiretinal membranes in a Japanese population: the Hisayama study. Graefes Arch Clin Exp Ophthalmol 2003; 241: 642-646.

7 McCarty DJ, Mukesh BN, Chikani V, Wang JJ, Mitchell P, Taylor HR et al. Prevalence and associations of epiretinal membranes in the visual impairment project. Am J Ophthalmol 2005; 140: 288-294.

8 You Q, Xu L, Jonas JB. Prevalence and associations of epiretinal membranes in adult Chinese: the Beijing eye study. Eye 2007 (in press).

9 Fraser-Bell S, Ying-Lai M, Klein R, Varma R. Prevalence and associations of epiretinal membranes in latinos: the Los Angeles Latino Eye Study. Invest Ophthalmol Vis Sci 2004; 45 1732-1736.

10 Mitchell P, Smith W, Chey T, Wang JJ, Chang A. Prevalence and associations of epiretinal membranes. The Blue Mountains Eye Study, Australia. Ophthalmology 1997; 104: 1033-1040.

11 Klein R, Klein BE, Wang Q, Moss SE. The epidemiology of epiretinal membranes. Trans Am Ophthalmol Soc 1994; 92: 403-425; discussion 425-30.

12 Kawasaki R, Wang JJ, Rochtchina E, Taylor B, Wong TY, Tominaga $\mathrm{M}$ et al. Cardiovascular risk factors and retinal microvascular signs in an adult Japanese population: the Funagata Study. Ophthalmology 2006; 113: 1378-1384.

13 Davis MD, Fisher MR, Gangnon RE, Barton F, Aiello LM, Chew EY et al. Risk factors for high-risk proliferative diabetic retinopathy and severe visual loss: early treatment diabetic retinopathy study report no.18. Invest Ophthalmol Vis Sci 1998; 39: 233-252.

14 Klein R, Davis MD, Magli YL, Segal P, Klein BE, Hubbard L. The Wisconsin age-related maculopathy grading system. Ophthalmology 1991; 98: 1128-1134.

15 Whitworth JA. 2003 World Health Organization (WHO)/ International Society of Hypertension (ISH) statement on management of hypertension. J Hypertens 2003; 21: 1983-1992.
16 Alberti KG, Zimmet PZ. Definition, diagnosis and classification of diabetes mellitus and its complications. Part 1: diagnosis and classification of diabetes mellitus provisional report of a WHO consultation. Diabet Med 1998; 15: 539-553.

17 Ahmad OB, Boschi-Pinto C, Lopez AD, Murray CJL, Lozano R, Inoue M. Age Standardization of Rates: a New WHO Standard. GPE Discussion Paper Series: no. 31. World Health Organization: Geneva, 2000.

18 Sekikawa A, Eguchi H, Tominaga M, Igarashi K, Abe T, Manaka $\mathrm{H}$ et al. Prevalence of type 2 diabetes mellitus and impaired glucose tolerance in a rural area of Japan. The Funagata diabetes study. J Diabetes Complications 2000; 14: 78-83.

19 Hikichi T, Trempe CL. Risk of bilateral idiopathic preretinal macular fibrosis. Eye 1995; 9(Part 1): 64-66.

20 Foos RY, Kreiger AE, Forsythe AB, Zakka KA. Posterior vitreous detachment in diabetic subjects. Ophthalmology 1980; 87: 122-128.

21 Sebag J. Abnormalities of human vitreous structure in diabetes. Graefes Arch Clin Exp Ophthalmol 1993; 231: 257-260.

22 Wiznia RA. Posterior vitreous detachment and idiopathic preretinal macular gliosis. Am J Ophthalmol 1986; 102: 196-198.

23 Smiddy WE, Michels RG, Green WR. Morphology, pathology, and surgery of idiopathic vitreoretinal macular disorders. A review. Retina 1990; 10: 288-296.

24 Harada C, Mitamura Y, Harada T. The role of cytokines and trophic factors in epiretinal membranes: involvement of signal transduction in glial cells. Prog Retin Eye Res 2006; 25: 149-164.

25 Fraser-Bell S, Guzowski M, Rochtchina E, Wang JJ, Mitchell P. Five-year cumulative incidence and progression of epiretinal membranes: the Blue Mountains Eye Study. Ophthalmology 2003; 110: 34-40. 